\title{
artigo
}

Ferreira, C.F.S.; Gonçalves, L.X.R.; Oliveira, M.S.S.; Lira, P.F.; Pereira, D.C.R.; Lopes da Silva, C.R.

Percepção da mulher quanto a assistência na forma de parir

\section{Percepção da mulher quanto a assistência na forma de parir}

\author{
Women's percetion on assistance in the way of paring \\ Percepción de las mujeres sobre la asistencia em la valora de partir
}

\begin{abstract}
RESUMO
A gravidez e o parto são eventos importantes na vida das mulheres. Até o século 20, o parto acontecia em casa, um momento pessoal e privado, compartilhado com outras mulheres. Objetivo: analisar na literatura a percepção das mulheres quanto à assistência na forma de parir. Método: trata-se de uma revisão sistemática da literatura com metassíntese, com utilização do protocolo PRISMA. Foram utilizados os descritores em ciências da saúde (DeCS): "enfermagem", "assistência ao parto" e "tocologia". associados ao operador booleano AND, da seguinte forma "enfermagem" AND "assistência ao parto" AND "tocologia", sendo encontrados 32 artigos. Resultados: Através dos critérios de inclusão e exclusão a amostra foi composta por 6 trabalhos. Conclusão: foi possivel analisar que os profissionais de saúde, em especial os enfermeiros precisam dar todas as informações e atenção necessária, através de uma assistência centrada no respeito a particularidade de cada mulher, possibilitando assim, uma vivência menos traumática do parto.
\end{abstract}

DESCRITORES: Enfermagem; Assistência ao parto; Tocologia.

\section{ABSTRACT}

Pregnancy and childbirth are important events in women's lives. Until the 20th century, childbirth took place at home, a personal and private moment, shared with other women. Objective: to analyze in the literature the perception of women regarding care in the way they give birth. Method: this is a systematic review of the literature with metasynthesis, using the PRISMA protocol. The descriptors in health sciences (DeCS) were used: "nursing", "birth care" and "tocology". associated with the Boolean operator AND, as follows "nursing" AND "birth care" AND "tocology", 32 articles being found. Results: Through the inclusion and exclusion criteria, the sample consisted of 6 studies. Conclusion: it was possible to analyze that health professionals, especially nurses, need to give all the necessary information and attention, through care centered on respect for the particularity of each woman, thus enabling a less traumatic experience of childbirth.

DESCRIPTORS: Nursing; Childbirth assistance; Tocology.

\section{RESUMEN}

El embarazo y el parto son acontecimientos importantes en la vida de las mujeres. Hasta el siglo XX, el parto tuvo lugar en casa, un momento personal y privado, compartido con otras mujeres. Objetivo: analizar en la literatura la percepción de las mujeres acerca de la atención en la forma en que dan a luz. Método: se trata de una revisión sistemática de la literatura con metasíntesis, utilizando el protocolo PRISMA. Los descriptores en ciencias de la salud (DeCS) fueron utilizados: "enfermería", "cuidado del nacimiento" y "tocología", asociados con el operador booleano Y, como sigue "enfermería" Y "cuidado del nacimiento" Y "tocología", 32 artículos encontrados. Resultados: A través de los criterios de inclusión y exclusión, la muestra consistió en 6 estudios. Conclusión: fue posible analizar que los profesionales de la salud, especialmente las enfermeras, necesitan dar toda la información y atención necesarias, a través de la atención centrada en el respeto de la particularidad de cada mujer, permitiendo así una experiencia menos traumática de parto.

DESCRIPTORES: Enfermería; Asistencia para el parto; Tocología.

RECEBIDO EM: 15/03/2021 APROVADO EM: 22/06/2021

\section{Carolina Francisca da Silva Ferreira}

Enfermeira, Pós-graduanda em Saúde da Família - FAVENI.

ORCID: 0000-0003-1955-0005 


\section{Laudilina Xavier Rocha Gonçalves}

Enfermeira, Pós-graduanda em Saúde da Família - FAVENI.

ORCID: 0000-0002-2352-7054

\section{Maria do Socorro Santos de Oliveira}

Enfermeira, Especialista em Enfermagem Obstétrica, Docente de Enfermagem - UNIJUAZEIRO.

ORCID: 0000-0001-9392-2378

\section{Petrúcya Frazão Lira}

Enfermeira, Mestre em Ciências da Educação, Especialista em Gestão de Redes, Docente da UNIJUAZEIRO, Coordenadora da Escola Técnica do SUS Dr. Antonio Marchet Callou.

ORCID: 0000-0001-9539-066X

\section{Dayse Christina Rodrigues Pereira}

Enfermeira, Doutora em Ciência da saúde, pelo Centro Universitário Saúde do ABC-FMABC, Diretora da Rede Municipal de Saúde Mental, Pós Doutoranda em Ciência da Saúde pelo Centro Universitário Saúde do ABC-FMABC.

ORCID: 0000-0002-5719-3574

\section{Cicero Rafael Lopes da Silva}

Enfermeiro, Especialista em Enfermagem Dermatológica, Pós-graduando em Docência do Ensino Superior - UNIJUAZEIRO e em Gerontologia e saúde do idoso - FAVENI, Docente da graduação em Enfermagem - UNIJUAZEIRO.

ORCID: 0000-0001-8819-5380

\section{INTRODUÇÃO}

A gravidez e parto são acontecimentos marcantes na vida das mulheres. Até o século XX o parto era realizado em casa cujo se constituía de um momento pessoal e privado, partilhado com outras mulheres. A assistência a parição era de responsabilidade unicamente feminina, visto que somente as parteiras e mulheres de confiança da gestante, realizavam essa prática. Dessa forma os médicos só eram chamados quando surgiam complicações que as parteiras não conseguiam resolver ${ }^{1}$.

Todavia, com os progressos de estudos relacionados a procedimentos cirúrgicos e com a chegada de instrumentos indicados para tal ato, foram instituídas normas para a regulamentação da profissão das parteiras, por conseguinte a presença do médico gradualmente foi ganhando espaço na assistência na forma de parir, desta maneira houve um aumento da hospitalização do parto, que permitiu a entrada da medicalização e controle do ciclo gravídico puerperal diminuindo os índices de risco de sequelas, mortalidade materna, perinatal e neonatal. Ocorrendo assim, a transferência do protagonismo da mulher para equipe médica e assistentes ${ }^{1-2}$.
Desse modo, o parto que anteriormente era um evento privado e familiar, passou a ser executado em esfera pública, em instituições de saúde com intensas medicações e rotinas cirúrgicas, sob conduta dos médicos, passando a ser considerado assim um ato cirúrgico ${ }^{1}$.

No Brasil, segundo dados do Departamento de Informática do Sistema Único de Saúde (DATASUS) no ano de 2019 os índices de partos cesáreos chegaram a $56,34 \%$ sendo apenas $43,66 \%$ partos vaginais ${ }^{3}$.

Desta forma, o parto cesáreo que tem como objetivo inicial atender às necessidades médicas não resolvidas pelo parto vaginal, teve seu uso discutido no mundo inteiro em reflexão sobre novas técnicas cirúrgicas e assépticas ${ }^{4}$.

No que diz respeito aos procedimentos cirúrgicos, a cesárea é tão comum no Brasil que o país é líder mundial neste procedimento. Segundo a Organização Mundial da Saúde (OMS), a cesárea tornou-se uma "epidemia" nos últimos 20 anos, sendo recomendado que apenas $10 \%$ a $15 \%$ dos partos sejam realizados por esse método 5 .

Desta forma ocorre, a transformação de um acontecimento fisiológico, saudável, em um acontecimento patológico, devido a mudança de papéis no qual a mulher deixa de ser protagonista, e o médico passa a ocupar o seu lugar. Diante disto, a mulher perdeu sua privacidade e autonomia, sendo submetida a regras institucionais e práticas intervencionistas ${ }^{1-2}$.

Desta forma, diante do exposto no presente estudo, questiona-se: Qual a percepção da mulher quanto à assistência na forma de parir?

Nesse âmbito, percebe-se a necessidade de ampliar o conhecimento das mulheres com relação à assistência na forma de parir, identificando suas preferências e indicações, devido à supervalorização ao modelo biomédico. Desta forma, explicar à temática e analisar qual a assistência que tem sido prestada a essas mulheres, possibilitando traçar estratégias que favoreçam uma assistência de qualidade e segura ao binômio mãe-filho.

Assim, este estudo é de grande relevância para a assistência na forma de parir, visto que tem a necessidade de uma integralidade no cuidado à gestante, em virtude da fragilidade emocional da mesma ao decorrer do processo, tendo em conta que, nos últimos anos, os avanços científicos e tecnológicos relacionados à assistência na forma de parir contribuíram para a hospitalização, levando assim uma quebra do processo natural de tal evento. 
Diante disso, o objetivo do estudo foi analisar na literatura a percepção das mulheres quanto à assistência na forma de parir.

\section{MÉTOdO}

O estudo em questão, trata-se de uma revisão sistemática da literatura com metassíntese. As revisões sistemáticas têm por finalidade investigar cientificamente, acompanhando protocolos específicos para a obtenção de resultados com altos níveis de evidências. Esse método de pesquisa, é adequado para reunir as opiniões de um conjunto de documentos escrito por vários autores sobre determinada temática, sendo capaz de apresentar resultados idênticos ou contrários ${ }^{6-7}$.

O instrumento utilizado foi o protocolo PRISMA (Preferred Reporting Items for Systematic Reviews and Meta-Analyses) do qual é projetado para ajudar os autores a melhorar as revisões sistemáticas

\section{Figura 1- Fluxograma de eleições dos estudos.}

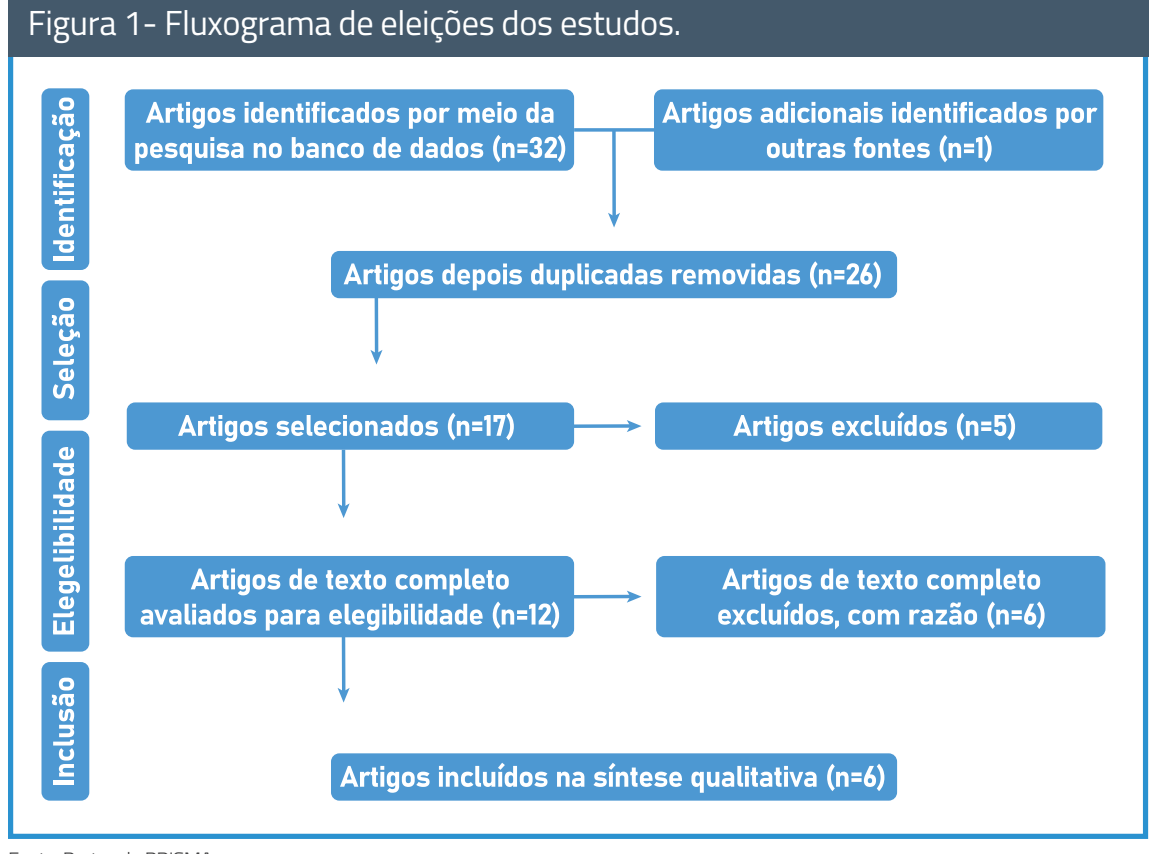

Fonte: Protocolo PRISMA. e relatórios de meta-análise. O foco está em ensaios clínicos randomizados, mas o PRISMA também pode ser usado como base para relatar revisões sistemáticas de outros tipos de estudos ${ }^{8}$.

A busca para alcançar a produção cientifica sobre a percepção da mulher quanto a assistência na forma de parir, foram utilizadas como fonte de busca a Biblioteca Virtual em Saúde (BVS) e as seguintes bases de dados: Scientific Electronic Library Online (SciELO), Literatura Latino-americana e do Caribe em Ciências da Saúde (LILACS) e Base de Dados em Enfermagem (BDENF). O levantamento das produções científicas ocorreu entre os meses de maio a outubro de 2020.

No decorrer da busca dos artigos, foram utilizados os descritores em ciências da saúde (DeCS): "enfermagem", "assistência ao parto" e "tocologia". associados ao operador booleano AND, da seguinte forma "enfermagem" AND "assistência ao parto" AND "tocologia”.

\section{Tabela 1 - Distribuição dos artigos quanto ao autor e ano de publicação, objetivo e principais resultados.}

\begin{tabular}{|c|c|c|c|}
\hline ANO & FONTE & AUTOR & OBJETIVOS \\
\hline 2016 & BDENF & $\begin{array}{l}\text { Melo DSA, Silva JMO, } \\
\text { Santos AA, Sanches } \\
\text { METL, Cavalcante KOR, } \\
\text { Jacintho, KS. }\end{array}$ & $\begin{array}{l}\text { Analisar a percepção da mulher relacionada } \\
\text { à assistência ao parto e identificar as difi- } \\
\text { culdades ocorridas durante esse processo. }\end{array}$ \\
\hline
\end{tabular}

\section{PRINCIPAIS RESULTADOS}

À assistência de saúde prestada durante o parto, é uma prática centrada ainda no modelo biomédico de assistência. 


\begin{tabular}{|c|c|c|}
\hline 2018 & LILACS & $\begin{array}{c}\text { Silva AB, Albuquerque } \\
\text { NLA, Carvalho ACS, Silva } \\
\text { RDM, Vicente CD. }\end{array}$ \\
\hline 2017 & SCIELO & $\begin{array}{l}\text { Ferreira LMS, Santos } \\
\text { ADF, Ramalho RCF, } \\
\text { Alves DA, Damasceno } \\
\text { SS, Figueiredo MFER, } \\
\text { Fernandes MRKGP, } \\
\text { Lemos ICS. }\end{array}$ \\
\hline 2016 & BDENF & $\begin{array}{c}\text { Ribeiro JF, Cruz AC, } \\
\text { Castro JDS, Luz VLES, } \\
\text { Monte NF, Barbosa MG. }\end{array}$ \\
\hline 2017 & LILACS & $\begin{array}{c}\text { Feitosa RMM, Pereira } \\
\text { RD, Souza TJCP, Freitas } \\
\text { RJM, Cabral SAR, Souza } \\
\text { LFF. }\end{array}$ \\
\hline 2019 & SCIELO & $\begin{array}{c}\text { Arik RM, Parada CMGL, } \\
\text { Tonete VLP, Sleutjes } \\
\text { FCM. }\end{array}$ \\
\hline
\end{tabular}

Silva $A B$, Albuquerque

Ferreira LMS, Santos ADF, Ramalho RCF,

Alves DA, Damasceno SS, Figueiredo MFER, Lemos ICS.
Identificar a percepção das mulheres acerca da assistência ao parto pela enfermeira obstetra.

Investigar a assistência de enfermagem ao trabalho de parto e parto, através da percepção das parturientes, buscando desse modo, contribuir para o aprimoramento do cuidado, uma vez que esse, para ser realizado, precisa da contribuição direta tanto do profissional, quanto do cliente.

Analisar a percepção de puérperas sobre a experiência com o parto normal e cesáreo.

Compreender, a partir da percepção das puérperas, os fatores que influenciam na escolha ao tipo de parto.

Apreender as percepções e expectativas de gestantes sobre o tipo de parto.
A falta de comunicação destitui da mulher o poder sobre seu próprio corpo, tornando-a mais passiva, impedindo assim, sua autonomia e participação ativa no processo de parir.

Deve-se reconhecer o valor do cuidado para as mulheres durante a atenção ao parto, ao mesmo tempo em que as práticas profissionais na assistência obstétrica devem ir ao encontro das necessidades individuais das mulheres, com respeito e sensibilidade.

Os fatores socioculturais, econômicos, históricos e emocionais têm se destacado como principal elemento para uma melhor aceitação da via de parto.

Dar voz às usuárias dos serviços de saúde, visando a identificação do seu conhecimento sobre os diversos fatores que envolvem a via de parto.

0 processo de decisão sobre a via de parto envolve, para além das preferências da gestante, as da família e as do médico.

\section{DISCUSSÃO}

Ao explorar as principais questões encontradas nos artigos contidos nesta revisão, foi possível identificar que vários autores têm a mesma concordância a respeito da percepção das gestantes acerca dos tipos de partos, dos seus sentimentos e da assistência prestada no cuidado durante o parto pelos profissionais, cujo tem que ser de forma individualizada, afetuosa, empática, segura e integral. Visando assim, uma assistência resolutiva e de qualidade.

Desta forma, baseando-se nos artigos selecionados, pesquisadores mostraram que, a gravidez é um período no qual a mulher se prepara para transformações na vida e para novas responsabilidades. É também nesse momento em que ela expõe seus sentimentos e receios relacionados ao parto ${ }^{10}$.

Á vista disso, no decorrer do parto, há fatores como ansiedade, dor, medo, hospitalização, dentre outros, que podem deixar as gestantes apreensivas, ocasionando a falta de controle dos momentos vivenciados. Contudo, as orientações, explicações e assistências prestadas por parte dos profissionais, são técnicas indicadas para superação destas dificuldades ${ }^{9-14}$.
Sendo assim, toda experiência vivenciada pelas mulheres durante esse momento, ficará firmemente marcada em sua memória, deste modo, o encorajamento e a confiança transmitida pelos profissionais da saúde em especial os enfermeiros, pode ter influência

\section{Desta forma, as}

gestantes serão

capazes de conhecer as

alternativas possíveis

na assistência, em

casos normais

e em situaçôes

que apareçam

complicações. marcante na diminuição da tensão emocional e ansiedade no parto, especialmente no momento que elas percebem que estão sendo protagonista do seu parto, podendo expor seus sentimentos, desejos e receios ${ }^{9-15-16}$.

Vale ressaltar que as boas condutas prestadas na assistência na forma de parir é a principal estratégia para ajudar o melhoramento do modelo obstétrico brasileiro, do qual é possível ofertar a essas mulheres assistência de qualidade baseando-se nas recomendações da Organização Mundial de Saúde (OMS) reiteradas pelas políticas de saúde no Brasi ${ }^{17-18 .}$

Estudos também revelam, uma deficiência no conhecimento das mulheres acerca dos tipos de partos, presença de contrações, dilatação, indicações de cesáreas, preparo físico e psíquico, entre outros, do qual todos esses conhecimentos devem ser iniciados precocemente nas consultas de pré-natal, pois é um elo de ligação para assistência na forma de parir. Desta forma, as gestantes serão capazes de conhecer as alternativas possíveis na assistência, em casos normais e em situações que apareçam complicações ${ }^{10}$.

Com isso, percebe-se a importância de uma educação em saúde na assistência, para mantê-las informadas a respeito de tudo que envolve o parto, seus sentimen- 


\section{artigo}

Ferreira, C.F.S.; Gonçalves, L.X.R.; Oliveira, M.S.S.; Lira, P.F.; Pereira, D.C.R.; Lopes da Silva, C.R.

Percepção da mulher quanto a assistência na forma de parir

tos e emoções, desta forma, elas terão uma assistência segura e de qualidade.

\section{CONCLUSÃO}

A partir das evidências científicas encontradas, percebeu-se que as mulheres carregam com si uma bagagem de sentimos e emoções, dos quais podem atrapalhar no momento de parir, interferindo assim no seu protagonismo na hora parto.

À vista disso, o presente estudo mostrou que existe uma escassez de informações oferecidas as mulheres acerca da assistência prestada na forma de parir, no qual essas informações tem grande importância para que possa ser evitado a elevação da taxa de partos cesáreos e consequentemen- te complicações na hora de parir.

Deste modo, os profissionais de saúde, em especial os enfermeiros que estão ao lado da paciente em todo contexto do cuidar, que assistem a essas mulheres precisam dar todas as informações e atenção necessária, através de uma assistência centrada no respeito a particularidade de cada mulher, possibilitando assim, uma vivência menos traumática do parto.

\section{REFERÊNCIAS}

1. Melo DSA, Silva JMO, Santos AA, Sanches METL, Cavalcante KOR, Jacintho, KS. Percepção da mulher quanto à assistência ao parto. Revista Enfermagem UFPE Online, v.10, supl.2, p. 81420, 2016.Disponivel em: 10.5205/reuol.6884-59404-2-SM1.1002 sup201616.

2. Silva $A B$, Albuquerque NLA, Carvalho ACS, Silva RDM, Vicente CD. Percepção da mulher acerca da assistência ao parto pela enfermeira obstetra. Enfermagem em foco, v.9, n.4, p. 28-22, 2018. Disponível em: https://doi.org/10.21675/2357707X.2018.v9.n4.1068.

3. DATASUS, Departamento de Informática do Sistema Único de Saúde. Tipo de parto dos nascidos vivos no Brasil. Disponivel em: http://tabnet.datasus.gov.br/cgi/deftohtm.exe?sinasc/cnv/nvuf def. Acessado em 17/06/2021.

4. Burin LG, Moron LMP, Charlo PB. Escolha do tipo de parto: avaliação do protagonismo da mulher. Revista Saúde Coletiva, v.10, n.57, 2020. Disponível em: https://doi.org/10.36489/saudecoletiva.2020v10i56p3545-3556.

5. Guimarães NM, Freitas VCS, Senzi CG, Gil GT, Lima LDSC, Frias DFR. Partos no sistema único de saúde (SUS) brasileiro: prevalência e perfil das partutientes. Brazilian Journal of Development, v.7, n.2, p. 11942-11958, 2021. Disponivel em: 10.34117/bjdv7n2-019.

6. Maria CBG, Ivan LMR. Revisão Sistemática Da Literatura: Conceituação, Produção e Publicação. Logeion Filosofia da Informação [Internet]. v. 6, n. 1, 2019. Disponível em: http://revista. ibict.br/fiinf/article/view/4835/4188.

7. Galvão MCB, Ricarte ILM. Revisão sistemática da literatura: Conceituação, produção e publicação. Logeion: Filosofia da Informação, vol. 06, n. 01, pag. 57-73, 15 de Set. 2019. DOI: https://doi.org/10.21728/logeion.2019v6n1.p57-73.

8. Moher D, Liberati A, Tetzlaff J, Altman DG, Galvão TF, Pansani TSA. Principais itens para relatar Revisões sistemáticas e Meta-análises: A recomendação PRISMA. Revista Epidemiologia e Serviços de Saúde, v.24, p.2, 2015. Disponível em: 10.5123/ S1679-49742015000200017.

9. Ferreira LMS, Santos ADF, Ramalho RCF, Alves DA, Damasceno SS, Figueiredo MFER, Fernandes MRKGP, Lemos ICS. Assistência de enfermagem durante o trabalho de parto e parto: a percepção da mulher. Revista Cubana de Enfermería, v.33, n.2, 2017. Disponivel em: http://revenfermeria.sld.cu/index.php/enf/ article/view/1102/263.
10. Ribeiro JF, Cruz AC, Castro JDS, Luz VLES, Monte NF, Barbosa MG. Experiência de parto: percepção das puérperas sobre o parto normal e cesáreo. Revista Enfermagem UFPE online, v.10, n.8, p.2801-8, 2016. Disponivel em: https://doi.org/10.5205/19818963-v10i8a11346p2801-2808-2016.

11. Feitosa RMM, Pereira RD, Souza TJCP, Freitas RJM, Cabral SAR, Souza LFF. Fatores que influenciam a escolha do tipo de parto na percepção das puérperas. Revista Online de Pesquisa Cuidado é Fundamental, v. 9, n. 3, p. 717-726, 2017. Disponível em: 10.9789/2175-5361.2017.v9i3.717-726.

12. Arik RM, Parada CMGL, Tonete VLP, Sleutjes FCM, Percepções e expectativas de gestantes sobre o tipo de parto. Revista Brasileira de Enfermagem, v.72, suppl.3, p. 46-54, 2019. Disponível em: http://dx.doi.org/10.1590/0034-7167-2017-0731.

13. Ribeiro JF, Cruz AC, Castro JDS, Luz VLES, Monte NF, Barbosa MG. Experiência de parto: percepção das puérperas sobre o parto normal e cesáreo. Revista Enfermagem UFPE online, v.10, n.8, p.2801-8, 2016. Disponivel em: https://doi.org/10.5205/19818963-v10i8a11346p2801-2808-2016.

14. Morais RF, Leite KNS, Silva SCR, Lima TNFA, Medeiros AM, Santos LMA. Expectativa das gestantes em relação ao parto normal e a cirurgia cesariana. Revista Temas em Saúde, v.18, n.1, p.414-427, 2018.

15. Rocha FAA, Fontenele FMC, Carvalho IR, Rodrigues IDCV, Sousa RA, Júnior ARF. Cuidado no parto e nascimento: percepção de puérperas. Revista Rene, v. 16, n. 6, p. 782-9, 2015. Disponivel em: 10.15253/2175-6783.2015000600003.

16. Almeida ARV, Alcântara DS, Araújo TT. Expectativas de gestantes em relação ao parto. Revista Interdisciplinar, v. 11, n. 1, p. 12-19, 2018. Disponível em: http://dx.doi. org/10.17648\%2F2317-5079.v11n1.1178.

17. Lira IMS, Melo SSS, Gouveia MTO, Feitosa VC, Guimarães TMM. Intervenção educacional para melhoria na assistência ao trabalho de parto normal. Revista electrónica trimestral de Enfermería, v.19, n. 2, p. 11, 2020. Disponivel em: https://doi. org/10.6018/eglobal.382581.

18. Gottems LBD, Carvalho EMP, Guilhem D, Pires MRGM. Boas práticas no parto normal: análise da confiabilidade de um instrumento pelo Alfa de Cronbach. Revista Latino-Americana de Enfermagem, v.26, 2018. Disponível em: 10.1590/15188345.2234.3000. 